PROCEEDINGS OF THE

AMERICAN MATHEMATICAL SOCIETY

Volume 127, Number 5, Pages 1301-1307

S 0002-9939(99)05004-2

Article electronically published on January 27, 1999

\title{
ON REAL QUADRATIC FUNCTION FIELDS OF CHOWLA TYPE WITH IDEAL CLASS NUMBER ONE
}

\author{
KEQIN FENG AND WEIQUN HU
}

(Communicated by David E. Rohrlich)

\begin{abstract}
Let $\mathbb{F}_{q}$ be the finite field with $q$ elements, $(2 \nmid q), k=\mathbb{F}_{q}(x), K=$ $k(\sqrt{D})$ where $D=D(x)=A(x)^{2}+a$ is a square-free polynomial in $\mathbb{F}_{q}[x]$ with $\operatorname{deg} A(x) \geq 1$ and $a \in \mathbb{F}_{q}^{*}$. In this paper several equivalent conditions for the ideal class number $h\left(O_{K}\right)$ to be one are presented and all such quadratic function fields with $h\left(O_{K}\right)=1$ are determined.
\end{abstract}

\section{INTRODUCTION}

Let $d=a^{2}+1 \geq 2$ be a square-free integer. R.A.Mollin [8] presented several equivalent conditions for the class number of real quadratic number field $K=\mathbb{Q}(\sqrt{d})$ to be one. S.Chowla conjectured that there are exactly 6 such fields with class number one. R.A.Mollin and H.C.Williams [9] proved this conjecture under the assumption of the Riemann hypothesis for $\zeta_{K}(s)$.

In this paper we will present an analogy of Mollin's sufficient conditions for ideal class number $h\left(O_{K}\right)$ of real quadratic function field $K$ to be one. We will show that the quadratic function field $K=k(\sqrt{D(x)})\left(k=\mathbb{F}_{q}(x), 2 \not \chi q\right)$ satisfying these conditions has to be of Chowla type: $D(x)=A(x)^{2}+a$ where $A(x) \in \mathbb{F}_{q}[x]$ and $a \in \mathbb{F}_{q}^{*}$. Since the Riemann hypothesis for function fields is true (A.Weil's theorem), we can determine all such quadratic function fields $K$ with $h\left(O_{K}\right)=1$.

\section{Preliminary Lemmas}

A systematic research on quadratic the function fields was initiated by E.Artin [1] in 1924, who gave the analytic formula for the class number (see section 4 ) and made a small table of class numbers. Let $k=\mathbb{F}_{q}(x)$ be the rational function field $(2 \not \chi q), D=D(x)$ a square-free polynimial in $\mathbb{F}_{q}[x]$ with $\operatorname{deg} D \geq 1, \operatorname{sgn} D$ the leading coefficient of the polynomial $D(x)$. Without loss of generality we can assume $\operatorname{sgn} D=1$ or $g$ where $g$ is a fixed generator of cyclic group $\mathbb{F}_{q}^{*}$. The quadratic function field $K=k(\sqrt{D})$ is called (by E.Artin) real if $2 \mid \operatorname{deg} D$ and $\operatorname{sgn} D=1$ since $\sqrt{D} \in k_{\infty}=\mathbb{F}_{q}\left(\left(\frac{1}{x}\right)\right)$ (the completion of $k$ at the infinite prime divisor $\infty=\left(\frac{1}{x}\right)$ ). Otherwise $K$ is called imaginary.

Received by the editors August 20, 1997.

1991 Mathematics Subject Classification. Primary 11R11, 11R29.

Key words and phrases. Quadradic field, function field, class number.

Research supported by the Natural Science Foundation and the National Educational Committee of China. 
Let $O_{K}$ be the integral closure of $\mathbb{F}_{q}[x]$ in $K, h\left(O_{K}\right)$ the ideal class number of $O_{K}$. J.R.C.Leitzel, M.L.Madan, C.S.Queen and R.E.MacRae $[5,6,7]$ determined all imaginary quadratic function fields $K$ (even for the case $2 \mid q$ ) with $h\left(O_{K}\right)=1$. For the real case, we can ask the following question as an analogy of a Gauss conjecture: Are there infinitely many of real quadratic function fields $K$ with $h\left(O_{K}\right)=1$ for any fixed $q$ ?

Let $K=k(\sqrt{D})$ be a real function field, $\operatorname{deg} D=2 d \geq 2$. The completion of $k=\mathbb{F}_{q}(x)$ at $\infty=\left(\frac{1}{x}\right)$ is the power series field $k_{\infty}=\mathbb{F}_{q}\left(\left(\frac{1}{x}\right)\right)$. Each element $0 \neq a \in k_{\infty}$ has the unique $\left(\frac{1}{x}\right)$-adic expansion

$$
a=\sum_{i=m}^{\infty} c_{i}\left(\frac{1}{x}\right)^{i}, \quad c_{i} \in \mathbb{F}_{q}, \quad c_{m} \neq 0 .
$$

Let $v_{\infty}(a)=m$, and $v_{\infty}(0)=\infty \cdot v_{\infty}$ is an extension of $\left(\frac{1}{x}\right)$-adic exponential valuation of $k$. Since $\sqrt{D} \in k_{\infty}, K$ is a subfield of $k_{\infty}$, and the restriction of $v_{\infty}$ to $K$ is an exponential valuation of $K$. The galois group Gal $(K / k)=\{1, \sigma\}$ where $\sigma(A+B \sqrt{D})=A-B \sqrt{D}(\mathrm{~A}, \mathrm{~B} \in k)$. We know that $O_{K}=\mathbb{F}_{q}[x] \oplus \mathbb{F}_{q}[x] \sqrt{D}$, and the unit group $U_{K}$ of $O_{K}$ is $\mathbb{F}_{q}^{*} \times\langle\varepsilon\rangle$ where $\varepsilon$ is a generator of the free part of $U_{K}$. Let $N=N_{K / k}$ be the norm mapping for $K / k$. Since $N(\alpha \varepsilon)=\alpha^{2} N(\varepsilon)$ for $\alpha \in \mathbb{F}_{q}^{*}$, we can assume $N(\varepsilon)=1$ or $g$. Since $N(\varepsilon)=\varepsilon \cdot \sigma(\varepsilon) \in \mathbb{F}_{q}^{*}$, we have $0=v_{\infty}(N(\varepsilon))=v_{\infty}(\varepsilon)+v_{\infty}(\sigma(\varepsilon))$, and $\varepsilon$ is determined by the condition $v_{\infty}(\varepsilon)<0$ up to factor $( \pm 1)$. We call this $\varepsilon$ the fundamental unit of $K$.

At the first step, we give a criterion for $h\left(O_{K}\right)=1$. For each ideal $\mathfrak{a} \neq(0)$, the order of the finite quotient ring $O_{K} / \mathfrak{a}$ is a $q$-th-power. If $\left|O_{K} / \mathfrak{a}\right|=q^{m}$, the degree of $\mathfrak{a}$ is defined by $\operatorname{deg} \mathfrak{a}=m \geq 1$. In [4] we showed that the Minkowski constant for real quadratic function field $K=k(\sqrt{D})$ is $d-1$ which means that we have

Lemma 2.1. Let $K=k(\sqrt{D})$ be a real quadratic function field, $\operatorname{deg} D=2 d \geq$ 2. Then each fractional ideal class of $O_{K}$ contains an integral ideal $\mathfrak{a}\left(\subseteq O_{K}\right)$ with $\operatorname{deg} \mathfrak{a} \leq d-1$. Therefore $h\left(O_{K}\right)=1$ if and only if all prime ideals of $O_{K}$ with degree $\leq d-1$ are principal.

Next, we introduce a result of Xianke Zhang [10], who determined the 2-rank of the ideal class group of $K$ by calculating the number of ambiguous ideal classes.

Lemma 2.2. Let $K=k(\sqrt{D})$ be a real quadratic function field, $C(K)$ the ideal class group of $O_{K}, r=\operatorname{dim}_{\mathbb{F}_{2}} C(K) / C(K)^{2}$ the 2-rank of $C(K)$, $m$ the number of monic irreducible polynomial factors of $D=D(x)$. Then $r=m-2$ if $D$ has an irreducible factor with odd degree, $r=m-1$ otherwise. Therefore $2 \nmid h\left(O_{K}\right)=\left|C_{K}\right|$ if and only if

(I) $D$ is irreducible, or

(II) $D=P_{1} P_{2}$ where $P_{1}$ and $P_{2}$ are irreducible polynomials with odd degree.

Remark 2.3. Let $\varepsilon$ be the fundamental unit of $K$. For the case (I) of lemma 2.2, E.Artin [1] proved $N(\varepsilon)=g$. For the case (II) we have $N(\varepsilon)=1$.

Proof. Let $\varepsilon=A+B \sqrt{D}\left(A, B \in \mathbb{F}_{q}[x]\right), P$ be an irreducible factor of $D$ with odd degree. If $g=N(\varepsilon)=A^{2}-D B^{2}$, we have $A^{2} \equiv g(\bmod P)$ and $\left(\frac{g}{P}\right)=1$ where $\left(\frac{g}{P}\right)$ is the Legendre symbol. But $2 \nmid \operatorname{deg} P$ implies $\left(\frac{g}{P}\right)=-1$, so we have contradiction (for more information on Legendre and Jacobi symbol in polynomial case, see section 4). 


\section{Real QUAdRatiC FUnCtion fields of Chowla type}

In this section we give an analogy of Mollin's result ([8], theorem 1) for real function field $K=k(\sqrt{D})$. At first we need some lemmas.

Definition 3.1. Let $E \in \mathbb{F}_{q}[x], E \neq 0$. A solution $(X, Y)=(U, V)$ of the equation

$$
X^{2}-D Y^{2}=E,
$$

in $\mathbb{F}_{q}[x]$ is called trivial if $E=a M^{2}, a \in \mathbb{F}_{q}^{*}, M \in \mathbb{F}_{q}[x]$, and $M|U, M| V$.

Lemma 3.2. Let $\varepsilon=A+B \sqrt{D}$ be the fundamental unit of $K . A, B \in \mathbb{F}_{q}[x]$. If the equation $(*)$ has a non-trivial solution in $\mathbb{F}_{q}[x]$, then $\operatorname{deg} E \geq \operatorname{deg} A-2 \operatorname{deg} B$.

Proof. Let $(U, V)$ be a non-trivial solution of the equation $(*)$, then $V \neq 0$. We can assume that $(U, V)$ is a non-trivial solution with minimal $\operatorname{deg} V$. We have

$$
\begin{aligned}
N(\varepsilon) E & =N[(A+B \sqrt{D})(U \pm V \sqrt{D})] \\
& =(A U \pm D B V)^{2}-D(B U \pm A V)^{2} .
\end{aligned}
$$

We claim that both of the solutions $(X, Y)=(A U \pm B D V, B U \pm A V)$ are non-trivial. If one of them is trivial, them $E=a M^{2}, a \in \mathbb{F}_{q}^{*}$ and

$$
\left\{\begin{array} { r l } 
{ A U + D B V } & { \equiv 0 \quad ( \operatorname { m o d } M ) } \\
{ B U + A V } & { \equiv 0 \quad ( \operatorname { m o d } M ) }
\end{array} \quad \text { or } \quad \left\{\begin{array}{r}
A U-D B V \equiv 0 \quad(\bmod M) \\
B U-A V \equiv 0 \quad(\bmod M) .
\end{array}\right.\right.
$$

But

$$
\begin{gathered}
(1) \cdot B-(2) \cdot A \Rightarrow A^{2} V-D B^{2} V \equiv 0(\bmod M) \Rightarrow V \equiv 0(\bmod M), \\
(1) \cdot A-(2) \cdot B D \Rightarrow A^{2} U-D B^{2} U \equiv 0(\bmod \quad M) \Rightarrow U \equiv 0(\bmod M) .
\end{gathered}
$$

Therefore the solution $(U, V)$ is trivial. This contradiction implies that both of the $(X, Y)=(A U \pm D B V, B U \pm A V)$ are non-trivial solutions. Since $\operatorname{deg} V$ is minimal we know that

$$
\min \{\operatorname{deg}(B U+A V)), \operatorname{deg}(B U-A V)\} \geq \operatorname{deg} V .
$$

On the other hand,

$$
\begin{aligned}
\operatorname{deg} E & =\operatorname{deg}\left(U^{2}-D V^{2}\right)=\operatorname{deg}\left(B^{2} U^{2}-D B^{2} V^{2}\right)-2 \operatorname{deg} B \\
& =\operatorname{deg}\left(B^{2} U^{2}-A^{2} V^{2}+V^{2} N(\varepsilon)\right)-2 \operatorname{deg} B .
\end{aligned}
$$

If $\operatorname{deg} B U>\operatorname{deg} A V$, then $\operatorname{deg} E=\operatorname{deg}\left(B^{2} U^{2}\right)-2 \operatorname{deg} B>\operatorname{deg}\left(A^{2} V^{2}\right)-2 \operatorname{deg} B>$ $2 \operatorname{deg} A-2 \operatorname{deg} B$. If $\operatorname{deg} B U<\operatorname{deg} A V$, then $\operatorname{deg} A^{2} V^{2}>\operatorname{deg} B^{2} U^{2}, \operatorname{deg} A^{2} V^{2}>$ $\operatorname{deg}\left(V^{2} N(\varepsilon)\right)$, therefore $\operatorname{deg} E=\operatorname{deg}\left(A^{2} V^{2}\right)-2 \operatorname{deg} B \geq 2 \operatorname{deg} A-2 \operatorname{deg} B$. Finally, if $\operatorname{deg} B U=\operatorname{deg} A V$, then $\max (\operatorname{deg}(B U+A V), \operatorname{deg}(B U-A V))=\operatorname{deg} A V$. From (3) we know that $\operatorname{deg}\left(B^{2} U^{2}-A^{2} V^{2}\right) \geq \operatorname{deg} A V^{2}$ and from (5) we have $\operatorname{deg} E \geq$ $\operatorname{deg} A V^{2}-2 \operatorname{deg} B \geq \operatorname{deg} A-2 \operatorname{deg} B$.

Q.E.D.

Lemma 3.3. Let $f$ be a positive integer. The following two conditions are equivalent.

(1) For each monic irreducible polynomial $P$ in $\mathbb{F}_{q}[x]$ with $\operatorname{deg} P \leq f$, we have $\left(\frac{D}{P}\right)=-1$ (which means that $P$ is inert in $\left.K\right)$.

(2) For each polynomial $A$ and irreducible polynomial $P$ in $\mathbb{F}_{q}[x]$ satisfying $\operatorname{deg} A<\operatorname{deg} P \leq f($ we assume $\operatorname{deg} 0=-\infty)$ we have $A^{2}-D \not \equiv 0(\bmod P)$.

Proof. It is obvious. 
Remark 3.4. The conditions in lemma 3.3 can be satisfied only for $f \leq d-1$ ( $2 d=$ $\operatorname{deg} D)$ since there exists a monic irreducible polynomial $P$ such that $\operatorname{deg} P \leq d$ and $\left(\frac{D}{P}\right) \neq-1$.

Proof. $D$ can always be expressed as $D=A^{2}+B$ with $\operatorname{deg} A=d$ and $\operatorname{deg} B \leq d-1$. If $\operatorname{deg} B \geq 1$, we choose $P$ as an irreducible factor of $B$, then $D \equiv A^{2}(\bmod P)$ and $\left(\frac{D}{P}\right) \neq-1$. If $B=b \in \mathbb{F}_{q}^{*}$, it is easy to see that there exists $a \in \mathbb{F}_{q}$ such that $a^{2}+b$ is a square in $\mathbb{F}_{q}$. Then $D=A^{2}+b \equiv a^{2}+b(\bmod A-a)$. We choose $P$ as an irreducible factor of $A-a$. Then $D \equiv a^{2}+b(\bmod P)$ and $\left(\frac{D}{P}\right) \neq-1$.

Now we come to our main result which shows that each condition in lemma 3.3 with $f=d-1$ is equivalent to $h\left(O_{K}\right)=1$ and $K$ is of Chowla type.

Theorem 3.5. Let $K=k(\sqrt{D})$ be a real quadratic function field, $\operatorname{deg} D=2 d \geq 2$, $k=\mathbb{F}_{q}(x), 2 \nmid q$. The following conditions are equivalent to each other.

(1) For any monic irreducible polynomial $P$ in $\mathbb{F}_{q}[x]$ with $\operatorname{deg} P \leq d-1$, we have $\left(\frac{D}{P}\right)=-1$.

(2) For any $A \in \mathbb{F}_{q}[x]$ and irreducible polynomial $P \in \mathbb{F}_{q}[x]$ satisfying $\operatorname{deg} A<$ $\operatorname{deg} P \leq d-1$ we have $D-A^{2} \not \equiv 0(\bmod P)$.

(3) For any $A \in \mathbb{F}_{q}[x]$ with $\operatorname{deg} A \leq d-1, D-A^{2}$ is either irreducible or a product of two irreducible polynomials with degree $d$.

(4) $h\left(O_{K}\right)=1$ and $K$ is of Chowla type: $D=A^{2}+b, A \in \mathbb{F}_{q}[x], b \in \mathbb{F}_{q}^{*}$.

Proof. $(1) \Longleftrightarrow(2)$ : By lemma 3.3 .

$(2) \Longrightarrow(3)$ : If $\operatorname{deg} A \leq d-1$ and $D-A^{2}$ has an irreducible factor $P$ with $\operatorname{deg} P \leq$ $d-1$, we can assume that $\operatorname{deg} A<\operatorname{deg} P$ by replacing $A$ if necessary by its least residue $\bmod P$. Therefore $\operatorname{deg} A<\operatorname{deg} P \leq d-1$ and $D-A^{2} \equiv 0(\bmod P)$ which contradicts (2).

$(3) \Longrightarrow(2):$ If $\operatorname{deg} A<\operatorname{deg} P \leq d-1$ and $D-A^{2} \equiv 0(\bmod P)$, then $D-A^{2}$ has the irreducible factor $P$ with $\operatorname{deg} P \leq d-1$ which contradicts (3).

$(4) \Longrightarrow(1)$ : We have $\varepsilon=A+\sqrt{D}, \operatorname{deg} A=d \geq 1$. If $\operatorname{deg} P \leq d-1$ and $\left(\frac{D}{P}\right) \neq-1$, then $P$ either ramifies or splits in $O_{K}$. Thus we have a prime ideal $\mathfrak{p}$ in $O_{K}$ such that $\mathfrak{p} \cdot \sigma(\mathfrak{p})=P$. From $h\left(O_{K}\right)=1$ we know that $\mathfrak{p}$ is principal: $\mathfrak{p}=(U+V \sqrt{D})$, therefore $\sigma(\mathfrak{p})=(U-V \sqrt{D})$ and

$$
U^{2}-V^{2} D=c P \quad\left(c \in \mathbb{F}_{q}^{*}\right) .
$$

The solution $(X, Y)=(U, V)$ of the equation $X^{2}-D Y^{2}=c P$ is non-trivial since $E=c P$ does not have the form $a M^{2}$. By lemma 3.2 we have $\operatorname{deg} P \geq \operatorname{deg} A-0=d$ which contradicts $\operatorname{deg} P \leq d-1$.

$(1) \Longrightarrow(4): h\left(O_{K}\right)=1$ comes from lemma 2.1. Moreover, we have the expression $D=A^{2}+B$ with $\operatorname{deg} A=d$ and $\operatorname{deg} B \leq d-1, B \neq 0$. If $\operatorname{deg} B \geq 1$, then $B$ has an irreducible factor $P$ with $\operatorname{deg} P \leq d-1$. We have $\left(\frac{D}{P}\right) \neq-1$ which contracicts (1). Therefore $B \in \mathbb{F}_{q}^{*}$ and $K$ is of Chowla type.

Q.E.D.

Remark 3.6. In fact, we can say more for $D$ if $K=k(\sqrt{D})$ satisfies the conditions of theorem 3.5. From condition (4) we know $D=A^{2}-a, a \in \mathbb{F}_{q}^{*}$ and $\operatorname{deg} A=d$. From condition (1), (2), or (3) we know that $D$ has no irreducible factor $P$ with $\operatorname{deg} P \leq d-1$. Therefore either $D$ is irreducible (and $a$ is not a square in $\mathbb{F}_{q}^{*}$ ) or $D=P_{1} P_{2}$ where $P_{1}$ and $P_{2}$ are irreducible with $\operatorname{deg} P_{1}=\operatorname{deg} P_{2}=d$. Since $h\left(O_{K}\right)=1$, the lemma 2.2 implies $2 \not d$ in the case $D=P_{1} P_{2}$. From remark 2.3 we 
know that $N(\varepsilon)=N(A+\sqrt{D})=a$ is a square in $\mathbb{F}_{q}^{*}$. Therefore $a=b^{2}\left(b \in \mathbb{F}_{\|}^{*}\right)$ and $P_{1}, P_{2}=A \pm b$. Thus $D=A^{2}-a$ has very special property:

(I) $D=A^{2}-a$ is irreducible and $a$ is not a square in $\mathbb{F}_{q}^{*}$; or

(II) $D=(A+b)(A-b)=A^{2}-b^{2}$ and $A \pm b$ are irreducible with odd degree $d$.

Suppose that $K=k(\sqrt{D})$ is a real quadratic function field and $D$ satisfies the condition (I) or (II) of remark 3.6. If $D$ does not satisfy the condition (1) of theorem 3.5 , then $h\left(O_{K}\right)>1$ and $2 \nmid h\left(O_{K}\right)$ (lemma 2.2). The following theorem presents a better lower bound for $h\left(O_{K}\right)$.

Theorem 3.7. Suppose that $K=k(\sqrt{D})$ is a real quadratic function field and $D$ satisfies the condition (I) or (II) of remark 3.6. If there exists an irreducible $P$ such that $\operatorname{deg} P \leq d-1$ and $\left(\frac{D}{P}\right) \neq-1$, then $h\left(O_{K}\right) \geq\left\langle\frac{d}{\operatorname{deg} P}\right\rangle$ where $\langle\alpha\rangle$ denotes the smallest odd integer $n$ such that $n \geq \alpha$.

Proof. From the assumption and lemma 2.2 we known that $h\left(O_{K}\right)$ is odd. From $\left(\frac{D}{P}\right) \neq-1$ we know that $P O_{K}=\mathfrak{p} \cdot \sigma(\mathfrak{p})$ where $\mathfrak{p}$ is a prime ideal of $O_{K}$. Let $n$ be the order of the ideal class $[\mathfrak{p}]$, then $2 \not n \mid h\left(O_{K}\right)$ and $\mathfrak{p}^{n}$ is a principal ideal. Let $\mathfrak{p}^{n}=(U+V \sqrt{D}), U, V \in \mathbb{F}_{q}[x]$, then $U^{2}-V^{2} D=c P^{n}, c \in \mathbb{F}_{q}^{*}$. From $2 \nmid \not n$ we know that $(X, Y)=(U, V)$ is a non-trivial solution of the equation $X^{2}-D Y^{2}=c P^{n}$. Lemma 3.2 implies that $\operatorname{deg} P^{n} \geq d$. Therefore $h\left(O_{K}\right) \geq n \geq \frac{d}{\operatorname{deg} P}$. Since $2 \nmid h\left(O_{K}\right)$ we know that $h\left(O_{K}\right) \geq\left\langle\frac{d}{\operatorname{deg} P}\right\rangle$.

Q.E.D.

\section{Determination of all Real QUADratic Function Fields OF CHOWla TYPE With ClASS NUMBER ONE}

This task has essentially been done in [3] since the following theorem is proved by using the Weil theorem and the Riemann-Roch theorem.

Theorem 4.1 ([3]). Suppose that $k=\mathbb{F}_{q}(x), 2 \nmid q q, K=k(\sqrt{D})$ is a real quadratic function field, $D=A^{2}+a, a \in \mathbb{F}_{q}^{*}$, $\operatorname{deg} A=d \geq 1$. If $h\left(O_{K}\right)=1$, then $q=3$, $d \leq 4 ; q=5, d \leq 2$; or $q \geq 7, d=1$.

Proof. We rewrite the proof here for the reader's convenience. The argument is taken from [7], p. 424. We know that $h\left(O_{K}\right) R_{K}=h(K)$ where $R_{K}=-v_{\infty}(\varepsilon)=$ $-v_{\infty}(A+\sqrt{D})=d$ (the regulator of $K$ ) and $h(K)$ is the divisor class number of $K$ (=the order of the divisor class group of degree zero). The genus of $K$ is $g_{K}=d-1$.

Let $n=2 g_{k}-1, \bar{k}=\mathbb{F}_{q^{n}}(x)$. Then $\bar{K}=\bar{k}(\sqrt{D})$ is a function field over $\mathbb{F}_{q^{n}}$ and $g_{\bar{K}}=g_{K}=d-1$. Let $\bar{N}_{1}$ be the number of prime divisors of $\bar{K}$ with degree 1 . The Weil theorem implies that

$$
\bar{N}_{1} \geq q^{n}+1-2 g_{\bar{K}} \cdot q^{n / 2} .
$$

$\bar{K} / K$ is a constant extension of degree $n$. Each prime divisor of $\bar{K}$ with degree $e$ is a product of $(e, n)$ distinct prime devisors of $K$ with degree $e /(e, n)$ (see [2], p. 164). Therefore the prime divisors of $\bar{K}$ with degree one come from the prime divisors of $K$ with degree $e \mid n$. And for $e \mid n$, a prime divisor $\mathfrak{p}$ of $K$ with degree $e$ give $e(\leq n)$ prime divisors of $\bar{K}$ with degree one and $\operatorname{deg} \mathfrak{p}^{n / e}=n$. Therefore the number of integral divisors of degree $n$ in $K$ is at least $\bar{N}_{1} / n$. On the other hand, the Riemann-Roch theorem says that the dimension of a divisor class $C$ of degree $n=2 g_{K}-1$ in $K$ is

$$
d(C)=\operatorname{deg} C+1-g_{K}=g_{K}=d-1 .
$$


There are precisely $\left(q^{d(c)}-1\right) /(q-1)$ integral divisors in each class $C$ (see [2], p.64), and we have $h(K)$ divisor classes of degree $n$. Therefore

$$
\begin{aligned}
\frac{h(K)\left(q^{d-1}-1\right)}{q-1} & \geq \frac{\bar{N}_{1}}{n} \geq \frac{q^{n}+1-2(d-1) q^{n / 2}}{n} \\
& =\frac{q^{2 d-3}+1-2(d-1) q^{\frac{2 d-3}{2}}}{2 d-3},
\end{aligned}
$$

and

$$
h\left(O_{K}\right) \geq \frac{(q-1)\left(q^{2 d-3}+1-2(d-1) q^{\frac{2 d-3}{2}}\right)}{d(2 d-3)\left(q^{d-1}-1\right)} .
$$

A simple calculation shows that the right-hand side of $(*)$ is bigger than one if $q \geq 7, d \geq 2 ; q=5, d \geq 3$; or $q=3, d \geq 5$. This completes the proof of theorem 4.1.

For the case of $d=1$, we have $g_{K}=0$ and $h\left(O_{K}\right)$ is always one. The following result gives all real quadratic function fields of Chowla type with $h\left(O_{K}\right)=1$ beside the trivial case of $d=1$.

Theorem 4.2. Suppose that $k=\mathbb{F}_{q}(x), 2 \nmid q, K=k(\sqrt{D}), D=A^{2}+a, a \in \mathbb{F}_{q}^{*}, A$ is monic polynomial in $\mathbb{F}_{q}[x]$ and $\operatorname{deg} A=d \geq 2$. There are precisely following six such fields with $h\left(O_{K}\right)=1$ :

$$
\begin{array}{ll}
q=3, & D=A^{2}+1 \quad \text { with } \quad A=x^{3}-x \pm 1, \quad x^{2}+1, \quad x^{2} \pm x-1, \\
q=5, \quad D=x^{4}+2 .
\end{array}
$$

Proof. From theorem 4.1 we know that there are only following finite cases to be considered: $q=3,2 \leq d \leq 4$; and $q=5, d=2$. $D$ has to satisfy the condition (I) or (II) of remark 3.6.

(I) $D$ is irreducible, $D=A^{2}-a, a \in \mathbb{F}_{q}^{*}-\left(\mathbb{F}_{q}^{*}\right)^{2}$. In [3] a table of $h(K)$ is presented for all quadratic function fields $K=k(\sqrt{P})$ where $P$ is irreducible and $3 \leq \operatorname{deg} P \leq 8$ for $q=3 ; 3 \leq \operatorname{deg} P \leq 5$ for $q=5$, and $3 \leq \operatorname{deg} P \leq 4$ for $q=7,11$. From this table we find exactly six fields $K=k(\sqrt{P})$ mentioned in theorem 4.2 satisfying the condition (I) and $h\left(O_{K}\right)=\frac{h(K)}{d}=1$.

Our class number table was made by using the following analytic formula given by E.Artin [1]:

$$
h(K)=-\sum_{i=1}^{2 d-1} i \sigma_{i}(D)
$$

where

$$
\sigma_{i}(D)=\sum_{\substack{A \in \mathbb{F}_{q}[x] \\ \text { monic } \\(A, D)=1 \\ \operatorname{deg} A=i}}\left[\frac{D}{A}\right]
$$

and $\left[\frac{D}{A}\right]$ is the Jacobi symbol for polynomials in $\mathbb{F}_{q}[x]$ which is a natural analogy of the ordinary Jacobi symbol and has similar properties:

(1) $\left(\frac{M_{1} M_{2}}{N}\right)=\left(\frac{M_{1}}{N}\right)\left(\frac{M_{2}}{N}\right)$ if $M_{1}, M_{2} \in \mathbb{F}_{q}[x], N \in \mathbb{F}_{q}[x]$ is monic, and $\left(M_{1}, N\right)=$ $\left(M_{2}, N\right)=1$.

$(2)\left(\frac{a}{N}\right)=a^{\frac{|N|-1}{2}}=a^{\frac{q-1}{2} \operatorname{deg} N}$, if $a \in \mathbb{F}_{q}^{*}, N \in \mathbb{F}_{q}[x]$ is monic and $|N|=q^{\operatorname{deg} N}$. Particularly, $\left(\frac{g}{N}\right)=(-1)^{\operatorname{deg} N}$. 
(3) (Reciprocity law) $\left(\frac{M}{N}\right)\left(\frac{N}{M}\right)=(-1)^{\frac{|M|-1}{2} \cdot \frac{|N|-1}{2}}=(-1)^{\frac{q-1}{2} \operatorname{deg} M \cdot \operatorname{deg} N}$ if $M$ and $N$ are monic polynomials in $\mathbb{F}_{q}[x]$ and $(M, N)=1$.

Moreover, we know that $\sigma_{0}=1, \sigma_{2 d-1}=-q^{d-1}$ and

$$
\sigma_{2 d-i}=q^{d-i}\left[-\sigma_{i-1}+(q-1)\left(\sigma_{i-2}+\cdots+\sigma_{1}+\sigma_{0}\right)\right] \quad(2 \leq i \leq d) .
$$

Therefore we need to compute $\sigma_{i}(D)$ for $1 \leq i \leq d-1$ only.

For the condition (II), $D=M^{2}-b^{2}=(M+\bar{b})(M-b), b \in \mathbb{F}_{q}^{*}, 2 \nmid d=\operatorname{deg} M \geq 2$ and $M \pm b$ are irreducible. The only case we need to consider is $q=3$ and $d=3$. There is only one field: $D=\left(x^{3}-x+1\right)\left(x^{3}-x-1\right)$. For this field we have $\sigma_{0}=1, \sigma_{5}=-9$. The formula (2) gives $\sigma_{1}=-3$ and $\sigma_{2}=9$. Then from (3) we have $\sigma_{4}=15$ and $\sigma_{3}=-13$. Therefore from the analytic formula (1)

$$
h\left(O_{K}\right)=\frac{h(K)}{3}=-\frac{1}{3}(-3+18-39+60-45)=3 \neq 1 \text {. }
$$

This completes the proof of theorem 4.2.

\section{REFERENCES}

[1] E.Artin, Quadratische Körper in Gebiet der höhren Kongruenzen I,II, Math. Zeit. 19(1924), 154-246.

[2] M.Deuring, Lectures on the theory of algebraic functions of one variable, LN in Math,NO.314,Springer-Verlag,1973. MR 49:8970

[3] Keqin Feng and Shuling Sun, On class number of quadratic fields, Proceeding of First International Symposium on Algebraic Structures and Number Theory (1988,Hong Kong),Edited by S.P.Lam and K.P.Shum, World Scientific, 1990,pp. 88-133. MR 91m:11098

[4] Weiqun $\mathrm{Hu}$, On the Minkowski constant for function fields, preprint, 1997.

[5] J.R.C.Leitzel,M.L.Madan and C.S.Queen, Algebraic function fields with small class number,Jour. of Number Theory, 7(1975),11-27. MR 51:5561

[6] R.E.MacRae, On unique factorization in certain rings of algebraic functions, Jour. of Algebra, 17(1971),243-261. MR 42:7643

[7] M.L.Madan and C.S.Queen, Algebraic function fields of class number one, Acta Arith. 20(1972),423-432. MR 46:5287

[8] R.A. Mollin, Necessary and sufficient conditions for the class number of a real quadratic field to be one, and a conjecture of S.Chowla,Proc. Amer. Math. Soc. 102(1988),17-21. MR 89d:11098

[9] R.A. Mollin, and H.C.Williams, A conjecture of S.Chowla via the generalized Riemann Hypothesis, Ibid,102(1988),794-796. MR 89d:11090

[10] Xianke Zhang, Ambiguous classes and 2-rank of class group of quadratic function field,Jour. of China Univ. of Sci. \& Tech.,19(1987), 425-431. MR 89j:11115

Graduate School at Beijing, University of Science and Technology of China, P. O. Box 3908, Beijing 100039, People's Republic of China

The Fundamental Science Department, Nanjing Agriculture College, Nanjing 210038, People's Republic of China 